\title{
(1) open Trajectory of intensive treat-to-target disease modifying drug regimen in an observational study of an early rheumatoid arthritis cohort
}

\author{
Douglas White, ${ }^{1}$ Helen Pahau, ${ }^{1}$ Emily Duggan, ${ }^{1}$ Sanjoy Paul, ${ }^{2}$ Ranjeny Thomas ${ }^{1}$
}

To cite: White $\mathrm{D}$, Pahau $\mathrm{H}$, Duggan $\mathrm{E}$, et al. Trajectory of intensive treat-to-target disease modifying drug regimen in an observational study of an early rheumatoid arthritis cohort. BMJ Open 2013;3: 0003083

doi:10.1136/bmjopen-2013003083

- Prepublication history for this paper is available online. To view these files please visit the journal online (http://dx.doi.org/10.1136/ bmjopen-2013-003083)

SP and RT contributed equally.

Received 18 April 2013 Revised 3 June 2013 Accepted 24 June 2013

${ }^{1}$ Diamantina Institute, University of Queensland, Princess Alexandra Hospital, Ipswich Road,

Woolloongabba, Queensland, Australia

${ }^{2}$ Queensland Clinical Trials and Biostatistics Centre, School of Population Health, University of Queensland, Translational Research Institute, Princess Alexandra Hospital, Brisbane, Queensland, Australia

Correspondence to Professor Ranjeny Thomas; ranjeny.thomas@uq.edu.au

\section{ABSTRACT}

Objectives: Studies of early rheumatoid arthritis (RA) cohorts have analysed treatment response and prognostic factors at fixed time points. However, in treat-to-target protocols, therapeutic decision-making is dynamic and responsive to disease activity over time.

To determine when a minimal residual disease response target should be expected, our primary objective was to identify the time-dependent therapeutic response to combination disease modifying antirheumatic drugs (DMARDs) for 12 months. Our secondary objective determined factors affecting this response trajectory.

Design: Observational cohort.

Setting: Treat-to-target early RA clinic in Australian tertiary referral hospital.

Participants: We enrolled consecutive patients attending an early arthritis clinic with symptom duration less than 12 months, who were diagnosed with RA for the first time between 2004 and 2008. 101 met these eligibility criteria and data were available at baseline through 12 months.

Interventions: intensive DMARDs according to a treat-to-target protocol.

Primary and secondary outcome measures: We measured disease activity scores (DAS) at each visit, then analysed therapeutic response and associated factors in a time-dependent fashion over 12 months.

Results: The median DAS4vESR of 4.46 at baseline decreased 12 weeks later by $24 \%$, while the proportion with DAS4v $\leq 2.6$ increased $(p<0.01)$. DAS4v continued to decrease over 52 weeks. DAS4v reduction of at least -0.45 at 4 weeks was predictive of DAS4v at 28 and 52 weeks. Female gender, current smoking, primary education and an interaction between baseline weight and $C$ reactive protein (CRP) negatively impacted DAS4v reduction over 4 and 52 weeks. Time-varying effects of blood pressure, neutrophils, erythrocyte sedimentation rate and CRP also significantly influenced DAS4v over 52 weeks.

Conclusions: Time-dependent data suggest that the largest reduction of DAS4v to combination DMARDs occurs in the first month of therapy, and this predicts subsequent response. Variables known to impact longterm treatment response in RA also impacted early DAS4v response to combination DMARDs.

\section{ARTICLE SUMMARY}

\section{Article focus}

- Best-practice early rheumatoid arthritis (RA) treatment aims to achieve a target response. In clinical settings of many countries, first-line therapies are disease modifying antirheumatic drugs (DMARDs), including combination DMARDs.

- We followed an observational cohort for 12 months in a treat-to-target early RA clinic to identify the time-dependent therapeutic response to combination DMARDs for 12 months and factors affecting this response trajectory.

\section{Key messages}

- After initiation of combination disease modifying antirheumatic drugs (DMARDs), the largest reduction in disease activity score occurred in the first month, and its magnitude predicted subsequent response.

- Disease activity score over 12 months was influenced by female gender and current smoking, education level and an interactive effect of weight and either $C$ reactive protein or erythrocyte sedimentation rate.

- The data suggest clinical response to combination DMARDs may be more rapid than previously appreciated, and treatment response in the first month may have prognostic significance.

- These hypotheses require further testing in other cohorts.

\section{BACKGROUND}

Intervention with early combination disease modifying antirheumatic drug (DMARD) therapy favourably influences progression of rheumatoid arthritis (RA) independent of treatment in later years, suggesting that there is a 'window of opportunity' in which the disease process can be altered. ${ }^{12}$ Moreover, a good response at 6 months to tight disease control using methotrexate predicted outcome after 5 years of treatment in participants in the CAMERA study. ${ }^{3}$ The severity of 


\section{ARTICLE SUMMARY}

Strengths and limitations of this study

- Monthly observation allowed precise determination of timedependent therapeutic response and demonstrated an unexpectedly rapid response to combination disease modifying antirheumatic drugs (DMARDs).

- Standardised combination DMARD treat-to-target protocol.

- Real-life clinical setting with dynamic therapeutic decision-making.

- Observational cohort study limits conclusions that can be drawn regarding causality, without further testing in a randomised controlled trial.

- Relatively small cohort derived from a single centre, with treatment regimen determined within Australian prescribing context, and exclusions due to missing data limit generalisability.

- Number of participants limited by lack of baseline or 12-month follow-up data and may have introduced selection bias.

- Owing to incomplete radiographic data, factors associated with radiographic outcomes could not be determined.

disease varies in RA patients. In those with aggressive disease, damage to articular structures occurs early in the disease process: erosions were detected in $12.8 \%$ of patients after a median of 8 weeks in one study. ${ }^{4}$ Thus, early evidence and determinants of treatment response to a given regimen are critical, in order to channel patients at greatest risk of poor outcome to more intensive induction regimens or more expensive biological therapies within that window.

Studies of prognostic factors by statistical modelling have analysed disease progression outcomes including erosions, disease activity score (DAS28) and disability index as measured by Health Assessment Questionnaire (HAQ) at fixed time points-usually 6 or 12 months, with the earliest being 3 months-to determine treatment response and associated factors influencing this. Factors associated with poor radiological outcome include smoking, rheumatoid factor (RF) positivity, the presence of anticitrullinated peptide autoantibodies (ACPA), HLA-DR genotype, low socioeconomic status and bone oedema on MRI. ${ }^{5-9}$ However, poor outcome measured by $\mathrm{HAQ}$ was associated with high baseline disease activity or HAQ including RF, DAS28 score, tender and swollen joint counts, ESR and CRP. ${ }^{10}{ }^{11}$ However, in treat-to-target protocols, such as was used in the TICORA trial and which occur in real-life clinic settings, decision-making about dose and drugs is a dynamic process, responsive to the patient's disease activity over time. ${ }^{12}$ In many early arthritis protocols, including the current study, patients are treated and monitored intensively during the first 3-6 months, followed by a reduced visit frequency. Longitudinal analysis of all available data, while modelling the trajectories and drawing inferences on the significance of various risk factors, provides higher power and better insight into the dynamic process.

\section{AIMS}

In the current study, our primary objective was to identify the time-dependent therapeutic response in an observational study of combination DMARDs for 12 months in order to determine when a minimal residual disease response target should be expected. Our secondary objective was to determine factors affecting this response trajectory. We therefore gathered disease activity data at each treatment visit then analysed the disease activity response in a time-dependent fashion. We then determined factors which influenced this time-dependent response to an intensive DMARD regimen.

\section{METHODS}

We enrolled consecutive patients referred by general practitioners from a relatively socioeconomically disadvantaged catchment $(60 \%$ referrals of employed individuals working in manual industries) to an early arthritis clinic in a public teaching hospital, with symptom duration less than 2 years, who were diagnosed with RA for the first time between 2004 and 2008. Patients were selected for inclusion in the current study if data were available at baseline through 12 months; however, data were not required at every time point for inclusion. Two hundred and six patients were referred with possible RA and 101 patients met these eligibility criteria; 49 did not have RA, and 54 patients who met all other criteria were excluded as data were unavailable at 12 months. Of these, seven were seen once and diagnosed with RA then treated elsewhere, and the remainder were reviewed at least once but not at 12 months. All study participants met the American College of Rheumatology 1987 revised criteria for the classification of RA. ${ }^{13}$

Referrals from local general practitioners were triaged within 1 week, and patients were generally diagnosed within the next 4 weeks. Since full clinical and laboratory evaluation was available at the first visit to the early arthritis clinic, patients received combination methotrexate (MTX), sulfasalazine (SSZ) and hydroxychloroquine (HCQ) ${ }^{14}$ unless contraindicated, immediately after diagnosis and confirmation of RA by the treating rheumatologist. Treatment was intensified according to a response-driven step-up algorithm, as previously described, ${ }^{15}$ with remission as the target. ${ }^{16}{ }^{17}$ Briefly, criteria for dose escalation were either $>2$ swollen joints and abnormal ESR or CRP, or at least two of the following four criteria: morning stiffness $>30$ mins, pain or fatigue visual analogue scale (VAS) $>30 \mathrm{~mm}$, or $>2$ tender joints. The following medications were prescribed at baseline: MTX $10 \mathrm{mg} /$ week, folic acid $5 \mathrm{mg}$ /week, SSZ $500 \mathrm{mg}$ daily increasing by $500 \mathrm{mg}$ at weekly intervals to $1000 \mathrm{mg}$ twice daily, HCQ $200 \mathrm{mg}$ daily for 1 week then $400 \mathrm{mg}$ daily thereafter. Patients were seen at 4-weekly intervals and the MTX dose was escalated according to treatment response at a conservative rate by $5 \mathrm{mg}$ at each visit to a maximum of $25 \mathrm{mg}$ weekly. If 
disease remained active on this combination, SSZ was stopped, MTX reduced to $10 \mathrm{mg}$ weekly and leflunomide started at a dose of $20 \mathrm{mg}$ daily. MTX dose was titrated back to $25 \mathrm{mg}$ weekly, and if this combination failed and the Australian Pharmaceutical Benefit Scheme criteria were met, the patient started biologic therapy. Based on these criteria, $10 \%$ of patients in this setting started biologics per year. In general, the use of non-steroidal anti-inflammatory drugs and oral corticosteroids was minimised, but intra-articular or oral steroids could be administered at the discretion of the treating physician. Large joints were injected with $40-80 \mathrm{mg}$ DepoMedrol and smaller joints with $1 \mathrm{~mL}(5.7 \mathrm{mg})$ Celestone. Oral and intra-articular dosage of corticosteroids was recorded monthly.

Response to therapy was measured as follows: the four variable DAS28ESR (hereafter referred to as DAS4v) was used as an index of inflammatory control, ${ }^{18}$ and the mHAQ as an index of disability. ${ }^{19}$ Each index was calculated at each visit: baseline, and weeks $4,8,12,16,22$, 28, 36, 44 and 52.

Demographic details were ascertained by questionnaire and included: age at presentation, symptom duration, level of education, gender, current, ex-smokers and non-smokers and mHAQ. Patients completed VAS for pain, fatigue and their global assessment of disease. The 28 tender and swollen joint counts, height, weight and blood pressure (BP) were recorded by the clinical research nurse. Blood was collected at baseline for the analysis of ESR, CRP, LFT, FBC, RF and ACPA. ACPA were measured at Queensland Health Pathology using the anti-CCP2 ELISA (Axis-Shield) test, with the cut-off of six for a positive test.

Basic statistics were presented by number (\%) or mean (SD) or median (IQR), as appropriate. Five imputations for missing data on clinical, biochemical and score data were performed using Bayesian-Markov chain Monte Carlo multiple-imputation technique. Of those who met eligibility criteria for study inclusion, not all patients attended for all visits, however the patterns of missingness were random for all the study parameters. The consistency in the distributions of the five imputed data was checked for all study parameters. Given the skewed DAS and mHAQ scores, the medians and their 95\% CI are presented. The changes in these scores over the study period are presented by median and 95\% CI. Significance levels ( $p$ values) are based on the appropriate non-parametric test.

Generalised multivariate linear regression models with $\gamma$ distribution and identity link were used to identify the statistically significant $(p \leq 0.10)$ risk factors and their possible interaction effects on disease activity scores at week 4 of the study. The possible consistency in the effect sizes of the statistically significant risk factors (at week 4) were also assessed on the disease activity scores at week 12 of the study. Combining the 10 longitudinal measurements obtained over 1 year of the study, the time varying effects of individual risk factors on the disease activity scores were explored using generalised estimating equation regression approach with $\gamma$ distribution and identity link function under the assumption of 'unstructured' correlation structure.

\section{RESULTS}

Time-dependent therapeutic response to combination DMARDs for 12 months

One hundred and one patients were included in the study and 54 (of whom 7 were only seen once) were excluded due to missing 12 month follow-up data. The baseline characteristics of the included and the excluded patients are shown in table 1. Except for a lower systolic BP in excluded subjects, there were no significant differences between included and excluded subjects. All except four patients took at least two and up to three of the following DMARDs in combination during the 12 months study: methotrexate, sulfasalazine, hydroxychloroquine and leflunomide. These four patients took methotrexate monotherapy.

The median disease activity score at baseline was 4.46 for DAS4v (table 2). Four of the 12 patients with

\begin{tabular}{|c|c|c|}
\hline \multirow[t]{2}{*}{ Baseline variable } & \multicolumn{2}{|l|}{ Value } \\
\hline & $\begin{array}{l}\text { Included } \\
\text { patients } \\
(n=101)\end{array}$ & $\begin{array}{l}\text { Excluded } \\
\text { patients } \\
(n=54)\end{array}$ \\
\hline Female† & $60(59.4)$ & $44(81)$ \\
\hline Age (years) $\ddagger$ & 54 (12) & 48 (15) \\
\hline $\begin{array}{l}\text { Symptom duration } \\
\text { (months)§ }\end{array}$ & $12(5,12)$ & $6(4,12)$ \\
\hline \multicolumn{3}{|l|}{ Smoking } \\
\hline Current smokers & $26(25.7)$ & $8(15)$ \\
\hline Ex-smokers & $29(28.7)$ & $26(48)$ \\
\hline \multicolumn{3}{|l|}{ Education } \\
\hline Primary & & $5(14)$ \\
\hline Secondary & & $24(66)$ \\
\hline Tertiary & & $9(24)$ \\
\hline Weight $(\mathrm{kg}) \ddagger$ & $77.10(19.68)$ & $80(24)$ \\
\hline $\mathrm{SBP}(\mathrm{mm} \mathrm{Hg}) \ddagger$ & $127(15)$ & $120(17)^{*}$ \\
\hline $\mathrm{DBP}(\mathrm{mm} \mathrm{Hg}) \ddagger$ & $73(10)$ & $70(10)$ \\
\hline $\mathrm{RF} \dagger$ & $89(88.1)$ & $42(77)$ \\
\hline ACPAT & $51(50.5)$ & $9(36)$ \\
\hline ESR $(\mathrm{mm} / \mathrm{h}) \S$ & $25(12,46)$ & $16(10,34)$ \\
\hline CRP $(\mathrm{mg} / \mathrm{L}) \S$ & $9.7(19,39)$ & $6(2,12)$ \\
\hline Lymphocytes $\left(\times 10^{9} / \mathrm{L}\right) \ddagger$ & $1.94(0.67)$ & $2.1(1.3,2.5)$ \\
\hline Neutrophils $\left(\times 10^{9} / \mathrm{L}\right) \ddagger$ & $5.12(2.50)$ & $5(3,6.8)$ \\
\hline LFT (AST) (U/L)§ & $20.50(16.50,24.00)$ & $18.5(17,23)$ \\
\hline LFT (ALT) (U/L)§ & $19(14,27)$ & $19(14,23)$ \\
\hline \multicolumn{3}{|c|}{$\begin{array}{l}{ }^{*} \mathrm{p}=0.002 . \\
\dagger \text { Values are } \mathrm{n}(\%) . \\
\text { †Values are the median (SD). } \\
\text { §Values are the median (IQR). } \\
\text { ACPA, anticitrullinated peptide antibody; ALT, alanine } \\
\text { aminotransferase; AST, aspartate aminotransferase; CRP, C } \\
\text { reactive protein; DBP, diastolic blood pressure; ESR, erythrocyte } \\
\text { sedimentation rate; LFT, liver function test; RF, rheumatoid factor; } \\
\text { SBP, systolic blood pressure. }\end{array}$} \\
\hline
\end{tabular}




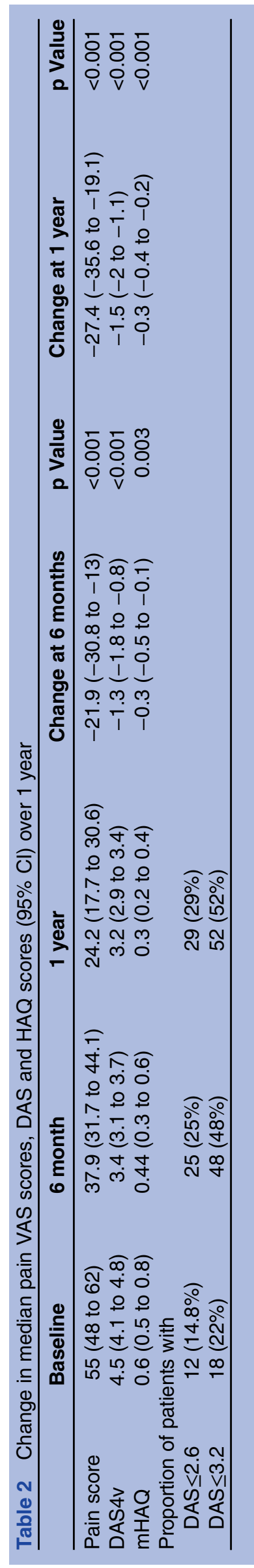

baseline DAS $\leq 2.6$ (minimal disease activity) were taking steroids prior to referral. There was a highly significant $(p<0.001)$ DAS reduction of $24 \%$ at 28 weeks (table 2 ). We also observed a significant increase in the proportion of patients with minimal residual, (DAS28 $\leq 2.6)$ and low disease (DAS28 $\mathrm{s} \leq 3.2)$ over the treatment period $(\mathrm{p}<0.01)$ (table 2). Consistent with this, the patients' pain scores improved highly significantly by $31 \%$ and $56 \%$ at the end of 6-month and 1-year of treatment, respectively. The improvement in mHAQ from baseline to 6 months, but not between 6 and 12 months of treatment, was significant. The average annual change was 0.30 units (table 2).

Analysis of the change in DAS4v over time showed a progressive reduction over 52 weeks, with the steepest drop between baseline and 4 weeks (figure 1). The median (95\% CI) of changes in DAS4v at 4, 28 and 52 weeks were $-0.45(-0.84,-0.07),-0.86 \quad(-1.30$, $-0.41)$ and $-1.35(-1.67,-1.03)$, respectively $(\mathrm{p}<0.01$ at week 52). The changing patterns of the distribution of DAS4v over time are evident from the density plots in figure 2. Although a significant shift in the distribution of DAS4v at 4 weeks from baseline is evident from the density plot, the distributions overlap at 4, 28 and 52 weeks.

Analysis of the individual components of the DAS over this period demonstrated that patient global score, swollen and tender joint counts all fell most steeply between baseline and 4 weeks (figure 2). While this was not the case for the fall in either ESR or CRP, similar steep falls in fatigue score, morning stiffness and physician global scores occurred between baseline and 4 weeks. Thus most measures of disease activity fell most rapidly in the first 4 weeks after DMARD initiation. In contrast, ESR fell for 3 months before reaching a plateau, while CRP fell progressively for 6 months.

\section{Factors affecting the response trajectory in early $\mathrm{RA}$ patients treated with combination DMARDs}

To determine whether the fall in DAS4v at 4 weeks predicted the DAS at 28 and 52 weeks, we first calculated that the median level of change in DAS4v at 4 weeks was -0.45 . This was clinically discriminatory: at 4 weeks, $52 \%$ had no change or an increase in DAS4v while $48 \%$ improved from baseline DAS4v. The number and proportion of patients receiving steroids is indicated in table 3. While baseline steroids impacted the likelihood of improvement at 4 weeks, this was not statistically significant $(69 \%$ of patients receiving steroids improved and $53 \%$ not receiving steroids improved; OR for improvement with steroids $1.95, \mathrm{p}=0.12$ ). Patients with reduction in DAS4v at 4 weeks of at least -0.45 were three times more likely (OR (95\% CI) 3.10 (1.2 to 8.0)) at 28 weeks and 17 times more likely (OR $(95 \%$ CI) 17.14 (4.52 to 64.94$)$ ) at 52 weeks to maintain the same or reduced DAS4v as achieved after 4 weeks of treatment. Univariate modelling of factors affecting outcome showed that female sex, smoking status and increasing 

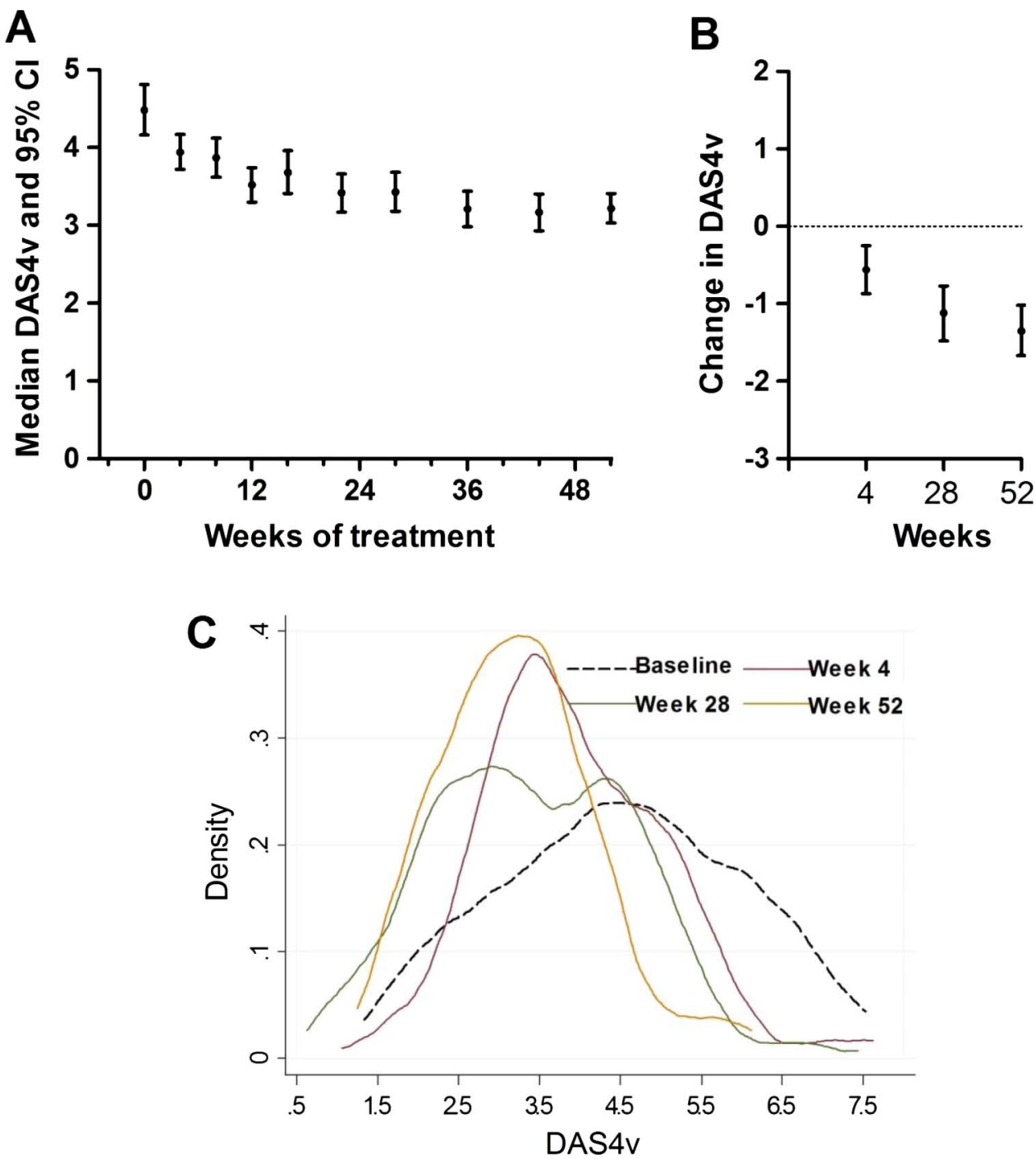

Figure 1 Distribution of DAS4v over the study period. (A) The median and $95 \% \mathrm{Cl}$ are plotted for each visit over the 52 week study period. (B) Changes in DAS4v over 4, 28 and 52 weeks are indicated. (C) The changing distribution in DAS4v in the sample is plotted at baseline, 4,28 and 52 weeks.

alanine aminotransferase (ALT) at baseline negatively affected DAS4v at 4 weeks, but these effects became less significant by 12 weeks (table 4 ). An interaction between baseline weight and CRP negatively affected DAS at both week 4 and 12. Patients taking steroid did not have a significantly different disease score, and symptom duration before RA diagnosis, anti-CCP or RF titre did not impact 4 -week DAS. The reduction in DAS4v at 4 weeks was significantly greater in patients with tertiary than with primary education.

Combining baseline characteristics and the longitudinal measurements obtained over 1 year, we explored the time-varying effects of individual risk factors on DAS4v in a univariate model (table 5). DAS4v over 52 weeks was again influenced by female gender and current smoking, and an interactive effect of weight and either CRP or ESR. Time varying effects of systolic and diastolic blood pressure, neutrophil counts, ESR and CRP also significantly influenced DAS4v observed over
52 weeks (table 4 ). At week 52, the largest reduction in DAS4v was observed in patients with tertiary education (3.57), compared with that observed among patients with secondary (2.56) or primary education (1.33). Symptom duration prior to diagnosis did not significantly influence DAS4v over 52 weeks. Over the course of the study, DAS4v was increased by 0.66 in those patients taking steroids $(\mathrm{p}<0.01)$. These data are, in keeping with the use of steroid in this study at the clinician's discretion, to provide additional control for disease activity that was not controlled by the DMARD protocol.

We found that the relationship between mHAQ and DAS4v for the cohort was significantly correlated at baseline, 4, 28 and 52 weeks $(p<0.001)$, with this correlation becoming progressively tighter over time as DAS and mHAQ fell. Thus, functional outcome after 1 year of early RA treatment is highly dependent on achievement of low disease activity. 
Figure 2 Variation in the disease activity parameters over the study period. The median and 95\% Cl are plotted for each visit over the 52-week study period for erythrocyte sedimentation rate, $\mathrm{C}$ reactive protein, tender joint count, swollen joint count, fatigue, morning stiffness, patient global and physician global scores.

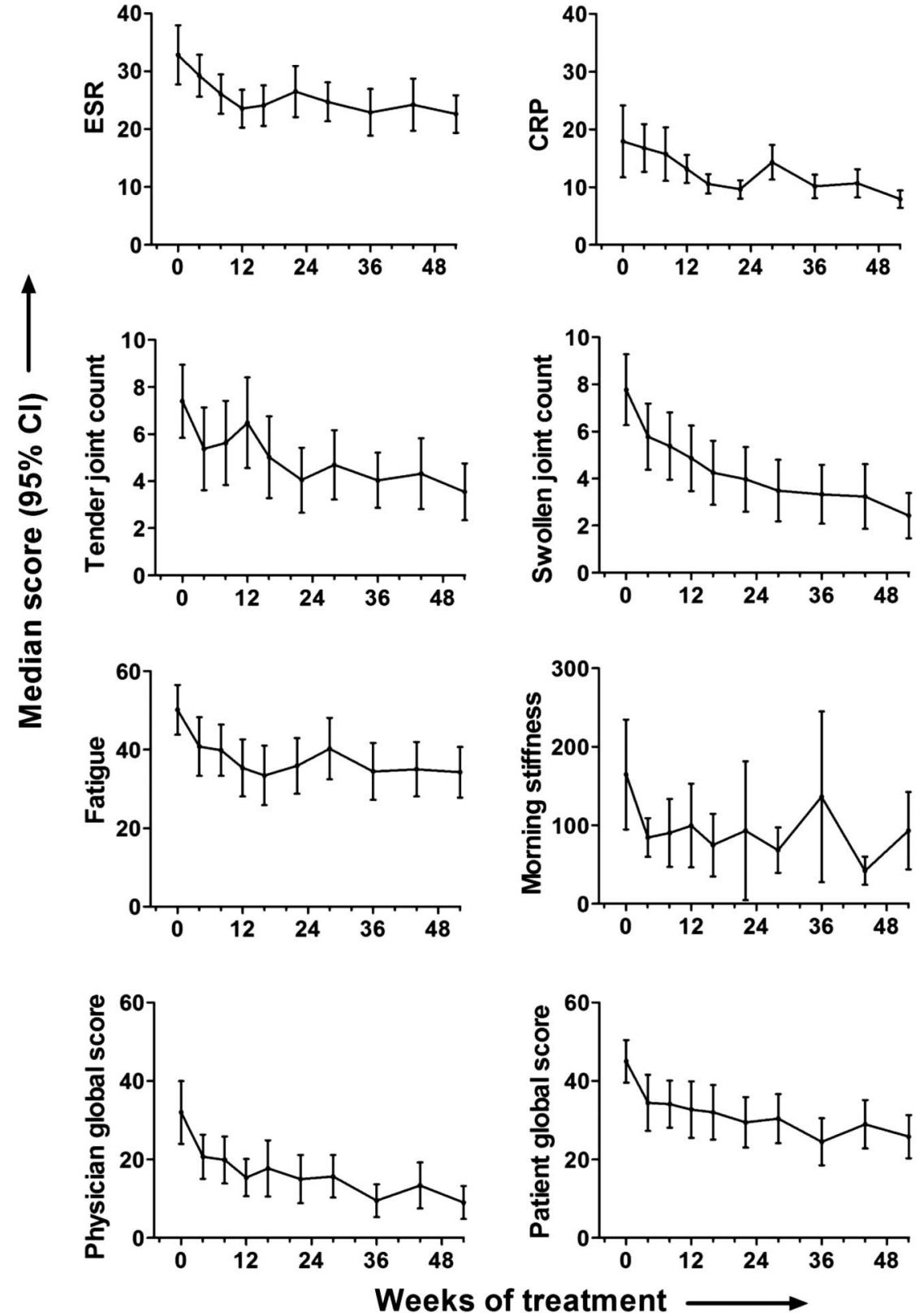

\section{DISCUSSION}

Our study describes the response of a group of patients with early RA to intensive conventional DMARD therapy in a time-dependent fashion over the first year. Baseline characteristics were in line with previous cohorts of patients with early RA. However, our baseline DAS scores were relatively low, reflecting our rapid triage and clinical and laboratory assessment of early arthritis referrals. Surprisingly, the time-dependent analysis of DAS response showed that the majority of disease activity

Table 3 Frequency of steroid use over the study

\begin{tabular}{|c|c|c|c|c|c|c|}
\hline \multirow[t]{2}{*}{ Treated with } & \multicolumn{6}{|c|}{ Study duration (weeks) } \\
\hline & $0(0)$ & 4 & 8 & 12 & 16 & 24 \\
\hline Oral steroid n (\%) & $16(15.8)$ & $17(16.8)$ & $14(13.9)$ & $11(10.9)$ & $1(1)$ & $1(1)$ \\
\hline IA steroid & $21(20.7)$ & $2(2)$ & $4(4)$ & $2(2)$ & 0 & 0 \\
\hline Any steroid & $37(36.7)$ & $19(18.8)$ & $18(17.8)$ & $13(12.9)$ & $1(1)$ & $1(1)$ \\
\hline Oral and IA steroid & $3(3)$ & $1(1)$ & $2(2)$ & 0 & 0 & 0 \\
\hline
\end{tabular}


Table 4 Variables influencing DAS scores at 4 and 12 weeks of study - univariate regression

\begin{tabular}{|c|c|c|c|c|}
\hline & \multicolumn{4}{|l|}{ DAS4v } \\
\hline & \multicolumn{2}{|l|}{ Week 4} & \multicolumn{2}{|c|}{ Week 12} \\
\hline & $\overline{\boldsymbol{\beta}}$ & p Value & $\overline{\boldsymbol{\beta}}$ & p Value \\
\hline \multirow{2}{*}{\multicolumn{5}{|c|}{ Smoking }} \\
\hline & & & & \\
\hline $\begin{array}{l}\text { Ex-smokers vs } \\
\text { non-smokers }\end{array}$ & -0.55 & 0.026 & -0.17 & 0.53 \\
\hline $\begin{array}{l}\text { Current } \\
\text { smokers vs } \\
\text { non-smokers }\end{array}$ & -0.80 & 0.003 & -0.42 & 0.10 \\
\hline LFT (ALT) & 0.03 & 0.01 & 0.04 & 0.63 \\
\hline Weight $\times$ CRP & 0.002 & 0.029 & 0.002 & 0.02 \\
\hline Oral or IA steroid & 0.11 & 0.67 & 0.01 & 0.98 \\
\hline Anti-CCP >6 & 0.0004 & 0.99 & 0.67 & 0.08 \\
\hline
\end{tabular}

Values are regression coefficient $(\beta)$ and $p$ value. Regression coefficient at each time point for $\mathrm{RF}=0$.

ALT, alanine aminotransferase; CRP, C reactive protein; LFT, liver function test; RF, rheumatoid factor.

measures fall most rapidly in the first 4 weeks after starting intensive DMARD treatment in this population. There was a subsequent slow and progressive reduction in DAS until week 52. This fall in DAS4v at 4 weeks appeared to be clinically meaningful, as it predicted the DAS at 28 and 52 weeks. This observation suggests that for patients who failed to respond within 4 weeks to combination DMARD treatment, few gains were made by continuing to apply the same DMARD treat-to-target algorithm for 6-12 months. This was reflected in the similar proportion of patients with minimal or low disease activity between 6 and 12 months. By this stage patients had progressed through the combination DMARD algorithm, for which the next step would be biologics. However, because their disease activity is minimal or low, they failed to qualify for biologics based on Australia's Pharmaceutical Benefit Scheme requirements (http://www.medicareaustralia. gov. au/provider/pbs/drugs2/rheumatoid.jsp)..$^{20}$ On the other hand, our data suggest the hypothesis that continued effort in applying a treat-to-target combination DMARD algorithm is likely to be effective over the ensuing months in patients who make a moderate response by week 4 . Our data further suggest that combination DMARDs act unexpectedly rapidly in this early RA population, as patients' use of steroids did not influence the reduction in DAS. In support of this conclusion regarding steroids, in a study of 61 patients with early RA treated according to a similar response-driven step-up combination DMARD algorithm, Proudman et $a l^{17}$ obtained an almost identical 6 month minimal disease activity rate (DAS28<2.6 in 29\%), despite infrequent use of corticosteroids.

The current study has a number of limitations. First, our interpretation that the magnitude of the fall in DAS4v after 1 month predicts 1 year outcome is limited by the observational study design. However, the question of whether outcome could be improved in patients with a minimal treatment response within 1 month could be tested in a randomised controlled trial comparing switch with biologic therapy with continued combination DMARDs. Second, this is a relatively small cohort derived from a single centre with referrals derived from a relatively socioeconomically disadvantaged catchment,

Table 5 Effects of time-varying risk factors individually on DAS28 scores over 1 year of study—univariate regression

\begin{tabular}{|c|c|c|c|}
\hline & \multicolumn{3}{|l|}{ DAS4v } \\
\hline & $\bar{\beta}$ & $95 \% \mathrm{Cl}$ & p Value \\
\hline Female & 0.45 & 0.09 to 0.81 & 0.014 \\
\hline Age & 0.001 & -0.13 to 0.02 & 0.82 \\
\hline \multicolumn{4}{|l|}{ Smoking } \\
\hline Ex-smokers vs non-smokers & -0.27 & -70 to 0.16 & 0.22 \\
\hline Current smokers vs non-smokers & -0.48 & -0.91 to -0.06 & 0.026 \\
\hline SBP & 0.10 & 0.08 to 0.20 & $<0.001$ \\
\hline DBP & 0.10 & 0.04 to 0.20 & 0.004 \\
\hline Lymphocyte & 0.04 & -0.09 to 0.17 & 0.55 \\
\hline Neutrophil & 0.16 & 0.10 to 0.22 & $<0.001$ \\
\hline ESR & 0.03 & 0.03 to 0.04 & $<0.001$ \\
\hline CRP & 0.02 & 0.01 to 0.02 & $<0.001$ \\
\hline LFT-AST & -0.004 & -0.01 to 0.003 & 0.25 \\
\hline LFT-ALT & -0.003 & -0.009 to 0.004 & 0.44 \\
\hline Weight $\times$ CRP & 0.002 & 0.001 to 0.003 & $<0.001$ \\
\hline Weight×ESR & 0.004 & 0.003 to 0.005 & $<0.001$ \\
\hline Oral or IA steroid & 0.66 & 0.34 to 0.99 & $p<0.01$ \\
\hline Anti-CCP>6 & 0.001 & -0.001 to 0.002 & 0.36 \\
\hline
\end{tabular}

Regression co-efficient at each time point for $\mathrm{RF}=0$.

ALT, alanine aminotransferase; CRP, C reactive protein; DBP, diastolic blood pressure; ESR, erythrocyte sedimentation rate; IA, intra-articular; LFT, liver function test; SBP, systolic blood pressure. 
with the treatment regimen determined within the Australian prescribing context. At the time of recruitment, 1987 ACR criteria were used to diagnose RA, which would have limited capacity to diagnose less severe patients. The number of participants was limited by lack of baseline or 12-month follow-up data and this may have introduced selection bias towards a more compliant group. The small sample size and number of exclusions due to incomplete data limit generalisability to other prescribing environments or clinical settings, and further studies are needed to test the generalisability of our findings. For example, it is possible that those excluded had a different disease trajectory due to differences risk for poor outcome or differences in adverse events. A subanalysis of the trajectory excluded patients was not possible because of the low number of paired baseline and 4-week DAS4v measurements in this group. On the other hand, there were no differences in the baseline characteristics of the excluded patients (except systolic BP). Furthermore, almost all factors associated with 4-week DAS4v response have been previously demonstrated to affect disease outcome in longer-term and larger studies. The strengths of this study are that it analyses real-world data, monthly observations allowed precise determination of time-dependent response, and patients received a standardised combination DMARD treat-to-target protocol, reducing the confounding effect of treatment decisions based on individual clinician preference.

The exploratory nature of the study in a relatively small sample could introduce false-positive associations. Although it is possible that the rapid 4-week response to the combination of DMARDs represents regression to the mean, the continued good response of these patients argues against this. Our data also are consistent with recent studies demonstrating that early good response to combination therapy (in the TEAR and RAPID 1 trials) is associated with a continued good response. ${ }^{21}{ }^{22}$ In these studies, rapid response was ascertained 12 weeks after initiation of combination therapy. By regression analysis, we identified female gender, current smoking, education level, ALT and an interaction between weight and CRP as significant determinants of disease activity over 4 and 52 weeks. Women, current smokers and low levels of education were found in several studies, including those of early RA, to achieve lower reductions in disease activity or remission. ${ }^{23-26}$ However, no study has previously determined that the impact of these variables may occur within weeks of starting treatment. The interaction between weight and inflammation in RA is intriguing and has been noted previously in insulin resistant states. ${ }^{27}$ In patients with active RA, those with high body mass index responded less well to infliximab. ${ }^{28}$ We also identified significant time-varying effects of blood pressure, gender, age, weight and inflammatory markers on disease activity. The interaction between disease activity and cardiovascular risk is well documented in RA, including early RA, and traditional cardiovascular risk factors may also impact the activity of inflammatory disease over time. ${ }^{15}{ }^{29-31}$ However, it is unknown whether control of cardiovascular risk factors can in turn impact inflammatory disease control.

In this study we were limited to analysis of disease and functional score, as radiographic data were not sufficiently complete to allow measurement of structural damage. However, this issue has been addressed by others, where biomarkers such as ACPA antibodies, RF, CRP and cartilage oligomeric matrix protein can add power to predictive models of bone erosion in early RA. ${ }^{32}$ In contrast, we found no impact of ACPA or RF on DAS. Our data confirm a strong relationship between disease activity and functional score that appears to strengthen over time, a finding that is supported by data from the BeST cohort. ${ }^{16}$ We would anticipate that functional disability would be minimised by early treatment with combination DMARDs as shown previously. ${ }^{33} 34$

Since they are traditionally thought to be slow acting, previous studies of DMARD monotherapy in early RA have not analysed time-dependent data from 4 weeks. Although it remains possible that a similar response might be observed in some patients starting DMARD monotherapy, we suggest this rapid response may be a unique feature of intensive combination DMARDs (with multiple mechanisms of action) initiation in early RA, which is the RA population most responsive to therapeutic intervention. ${ }^{35}$ The risks and benefits of intensive DMARD therapy (combinations allowing switching to achieve tight control) versus monotherapy in early RA deserve further study, considering inconsistent evidence to support combination DMARD therapy in RA. ${ }^{36} 37$ The need to identify patients with more aggressive disease prompted one group to undertake a trial of a stratified treatment plan based on the likelihood of persistent arthritis, with the aim of minimising over treatment and under treatment in early RA. ${ }^{38}$ Our data suggest the hypothesis that very early response to an intensive DMARD strategy that minimises under treatment predicts response for the first year.

Data from the ERAN study show that patients with moderate disease activity at 1 year are unlikely to achieve better control of their disease if the same protocol is continued, and a good response at 6 months in the CAMERA study predicted outcome at 5 years. ${ }^{3}{ }^{39}$ Our data, collected in a cohort of early RA patients with relatively low baseline DAS, likely reflect the trajectory of patients meeting criteria for RA early in disease, and which would be captured in organised clinical settings using the recently published new classification criteria. ${ }^{40}$

\section{CONCLUSIONS}

With the availability of increasing numbers of treatment options, application of strategies that identify early nonresponders to intensive DMARD combinations, has clear implications for treatment stratification within the 
window of opportunity. Time-dependent data suggest clinical response to combination DMARDs may be more rapid than previously appreciated, and treatment response in the first month may have prognostic significance. Confirmation in other cohorts will be required to determine the generalisability of this notion.

Contributors DW, SP, RT were involved in conception and design. DW, HP, ED contributed by acquiring, analysing and interpreting the data. DW, HP, ED, $\mathrm{SP}, \mathrm{RT}$ were involved in drafting the article or revising it critically for important intellectual content, and final approval before submission.

Funding The study was supported by NHMRC grants 351439 and 569938 . RT is supported by Arthritis Queensland and an ARC Future Fellowship.

Competing interests None.

Ethics approval Ethical approval for retrospective data analysis was obtained from the Metro South Human Research Ethics Committee.

Provenance and peer review Not commissioned; externally peer reviewed.

Data sharing statement No additional data are available.

Open Access This is an Open Access article distributed in accordance with the Creative Commons Attribution Non Commercial (CC BY-NC 3.0) license, which permits others to distribute, remix, adapt, build upon this work noncommercially, and license their derivative works on different terms, provided the original work is properly cited and the use is non-commercial. See: http:// creativecommons.org/licenses/by-nc/3.0/

\section{REFERENCES}

1. Mottonen $\mathrm{T}$, Hannonen $\mathrm{P}$, Leirisalo-Repo $\mathrm{M}$, et al. Comparison of combination therapy with single-drug therapy in early rheumatoid arthritis: a randomised trial. FIN-RACo trial group. Lancet 1999;353:1568-73.

2. Boers M, Verhoeven AC, Markusse HM, et al. Randomised comparison of combined step-down prednisolone, methotrexate and sulphasalazine with sulphasalazine alone in early rheumatoid arthritis. Lancet 1997;350:309-18.

3. Bakker MF, Jacobs JW, Welsing PM, et al. Early clinical response to treatment predicts 5-year outcome in RA patients: follow-up results from the CAMERA study. Ann Rheum Dis 2011;70:1099-103.

4. Machold KP, Stamm TA, Eberl GJ, et al. Very recent onset arthritisclinical, laboratory, and radiological findings during the first year of disease. J Rheumatol 2002;29:2278-87.

5. Harrison MJ, Tricker KJ, Davies L, et al. The relationship between social deprivation, disease outcome measures, and response to treatment in patients with stable, long-standing rheumatoid arthritis. J Rheumatol 2005;32:2330-6.

6. Manfredsdottir VF, Vikingsdottir T, Jonsson T, et al. The effects of tobacco smoking and rheumatoid factor seropositivity on disease activity and joint damage in early rheumatoid arthritis. Rheumatology (Oxford) 2006;45:734-40.

7. Kaltenhauser S, Pierer M, Arnold S, et al. Antibodies against cyclic citrullinated peptide are associated with the DRB1 shared epitope and predict joint erosion in rheumatoid arthritis. Rheumatology (Oxford) 2007;46:100-4.

8. Hetland ML, Ejbjerg B, Horslev-Petersen K, et al. MRI bone oedema is the strongest predictor of subsequent radiographic progression in early rheumatoid arthritis. Results from a 2-year randomised controlled trial (CIMESTRA). Ann Rheum Dis 2009;68:384-90.

9. Sanmarti R, Gomez-Centeno A, Ercilla G, et al. Prognostic factors of radiographic progression in early rheumatoid arthritis: a two year prospective study after a structured therapeutic strategy using DMARDs and very low doses of glucocorticoids. Clin Rheumatol 2007; $26: 1111-18$

10. Combe B, Cantagrel A, Goupille P. Predictive factors of 5-year health assessment questionnaire disability in early rheumatoid arthritis. J Rheumatol 2003;30:2344-9.

11. Wagner E, Ammer K, Kolarz G, et al. Predicting factors for severity of rheumatoid arthritis: a prospective multicenter cohort study of 172 patients over 3 years. Rheumatol Int 2007;27:1041-8.

12. Grigor $\mathrm{C}$, Capell $\mathrm{H}$, Stirling $\mathrm{A}$, et al. Effect of a treatment strategy of tight control for rheumatoid arthritis (the TICORA study): a single-blind randomised controlled trial. Lancet 2004;364:263-9.
13. Arnett FC, Edworthy SM, Bloch DA et al. The American Rheumatism Association 1987 revised criteria for the classification of rheumatoid arthritis. Arthritis Rheum 1988;31:315-24.

14. O'Dell JR, Haire CE, Erikson N, et al. Treatment of rheumatoid arthritis with methotrexate alone, sulfasalazine and hydroxychloroquine, or a combination of all three medications. N Engl J Med 1996;334:1287-91.

15. Hannawi S, Marwick TH, Thomas R. Inflammation predicts accelerated brachial arterial wall changes in patients with recent-onset rheumatoid arthritis. Arthritis Res Ther 2009;11:R51.

16. Van der Kooij SM, Allaart CF, Dijkmans BA, et al. Innovative treatment strategies for patients with rheumatoid arthritis. Curr Opin Rheumatol 2008;20:287-94.

17. Proudman SM, Keen HI, Stamp LK, et al. Response-driven combination therapy with conventional disease-modifying antirheumatic drugs can achieve high response rates in early rheumatoid arthritis with minimal glucocorticoid and nonsteroidal anti-inflammatory drug use. Semin Arthritis Rheum 2007;37: 99-111.

18. Wells G, Becker JC, Teng J, et al. Validation of the 28-joint Disease Activity Score (DAS28) and European League Against Rheumatism response criteria based on C-reactive protein against disease progression in patients with rheumatoid arthritis, and comparison with the DAS28 based on erythrocyte sedimentation rate. Ann Rheum Dis 2009;68:954-60.

19. Pincus T, Swearingen C, Wolfe F. Toward a multidimensional Health Assessment Questionnaire (MDHAQ): assessment of advanced activities of daily living and psychological status in the patient-friendly health assessment questionnaire format. Arthritis Rheum 1999;42:2220-30.

20. Lu CY, Williams KM, Day RO. Has the use of disease-modifying anti-rheumatic drugs changed as a consequence of controlled access to high-cost biological agents through the Pharmaceutical Benefits Scheme? Intern Med J 2007;37:601-6.

21. Van der Heijde D, Keystone EC, Curtis JR, et al. Timing and magnitude of initial change in disease activity score 28 predicts the likelihood of achieving low disease activity at 1 year in rheumatoid arthritis patients treated with certolizumab pegol: a post-hoc analysis of the RAPID 1 trial. J Rheumatol 2012;39:1326-33.

22. Curtis JR, McVie T, Mikuls TR, et al. Clinical response within 12 weeks as a predictor of future low disease activity in patients with early RA: results from the TEAR trial. J Rheumatol 2013;40: 572-8

23. Sokka T, Toloza S, Cutolo $\mathrm{M}$, et al. Women, men, and rheumatoid arthritis: analyses of disease activity, disease characteristics, and treatments in the QUEST-RA study. Arthritis Res Ther 2009;11:R7.

24. Saevarsdottir $\mathrm{S}$, Wallin $\mathrm{H}$, Seddighzadeh $\mathrm{M}$, et al. Predictors of response to methotrexate in early DMARD naive rheumatoid arthritis: results from the initial open-label phase of the SWEFOT trial. Ann Rheum Dis 2011;70:469-75.

25. Socioeconomic deprivation and rheumatoid disease: what lessons for the health service? ERAS Study Group. Early Rheumatoid Arthritis Study. Ann Rheum Dis 2000;59:794-9.

26. Vliet Vlieland TP, Buitenhuis NA, Van Zeben D, et al. Sociodemographic factors and the outcome of rheumatoid arthritis in young women. Ann Rheum Dis 1994;53:803-6.

27. Lopez-Bermejo A, Hernandez-Aguado I, Vera IJ, et al. Sex-specific, independent associations of insulin resistance with erythrocyte sedimentation rate in apparently healthy subjects. Thromb Haemost 2007;97:240-4.

28. Klaasen R, Wijbrandts CA, Gerlag DM, et al. Body mass index and clinical response to infliximab in rheumatoid arthritis. Arthritis Rheum 2011;63:359-64.

29. Bartoloni E, Alunno A, Luccioli F, et al. Atherosclerotic vascular damage and rheumatoid arthritis: a complex but intriguing link. Expert Rev Cardiovasc Ther 2010;8:1309-16.

30. Hannawi S, Haluska B, Marwick TH, et al. Atherosclerotic disease is increased in recent onset rheumatoid arthritis: a critical role for inflammation. Arthritis Res Ther 2007;9:R116.

31. Chen YS, Yan W, Geczy CL, et al. Serum levels of soluble receptor for advanced glycation end products and of S100 proteins are associated with inflammatory, autoantibody, and classical risk markers of joint and vascular damage in rheumatoid arthritis. Arthritis Res Ther 2009;11:R39.

32. Lindqvist $\mathrm{E}$, Eberhardt $\mathrm{K}$, Bendtzen $\mathrm{K}$, et al. Prognostic laboratory markers of joint damage in rheumatoid arthritis. Ann Rheum Dis 2005;64:196-201.

33. Makinen $\mathrm{H}$, Kautiainen $\mathrm{H}$, Hannonen $\mathrm{P}$, et al. Sustained remission and reduced radiographic progression with combination disease modifying antirheumatic drugs in early rheumatoid arthritis. J Rheumatol 2007;34:316-21. 
34. Hetland ML, Stengaard-Pedersen $\mathrm{K}$, Junker $\mathrm{P}$, et al. Radiographic progression and remission rates in early rheumatoid arthritis-MR bone oedema and anti-CCP predicted radiographic progression in the 5-year extension of the double-blind randomised CIMESTRA trial. Ann Rheum Dis 2010;69:1789-95.

35. Geletka R, St Clair EW. Treatment of early rheumatoid arthritis. Best Pract Res Clin Rheumatol 2003;17:791-809.

36. Moreland LW, O'Dell JR, Paulus HE, et al. A randomized comparative effectiveness study of oral triple therapy versus etanercept plus methotrexate in early aggressive rheumatoid arthritis: the treatment of Early Aggressive Rheumatoid Arthritis Trial. Arthritis Rheum 2012;64:2824-35.

37. Smolen JS, Landewe R, Breedveld FC, et al. EULAR recommendations for the management of rheumatoid arthritis with synthetic and biological disease-modifying antirheumatic drugs. Ann Rheum Dis 2010;69:964-75.

38. Claessen SJ, Hazes JM, Huisman MA, et al. Use of risk stratification to target therapies in patients with recent onset arthritis; design of a prospective randomized multicenter controlled trial. $B M C$ Musculoskelet Disord 2009;10:71.

39. Kiely $\mathrm{P}$, Walsh $\mathrm{D}$, Williams $\mathrm{R}$, et al. Outcome in rheumatoid arthritis patients with continued conventional therapy for moderate disease activity - the early RA network (ERAN). Rheumatology (Oxford) 2011;50:926-31.

40. Aletaha D, Neogi T, Silman AJ, et al. 2010 Rheumatoid arthritis classification criteria: an American College of Rheumatology/ European League against rheumatism collaborative initiative. Arthritis Rheum 2010;62:2569-81. 